\title{
TIMP-2*IGFBP7 as an auxiliary identification of successful discontinuation CRRT and prediction of renal recovery in critically ill patients: a case control study
}

yuanyuan xie ( $\nabla$ xie_yuanyuan516@126.com )

Renji Hospital, School of Medicine, Shanghai Jiaotong University https://orcid.org/0000-0001-62701935

Alexander Zarbock

department of anesthesiology, intensive care and pain medicine, university hosital munster

Alessandra Brendolan

Ospedale San Bortolo di Vicenza

Francesca Martino

Ospedale San Bortolo di Vicenza

Sara Samoni

Ospedale San Bortolo di Vicenza

Nicola Marchionna

Ospedale San Bortolo di Vicenza

Weixuan fan

internation renal research institute of vicenza

\section{Silvia De Rosa}

Ospedale San Bortolo di Vicenza

\section{Davide Giavarina}

Ospedale San Bortolo di Vicenza

Gregorio Romero-González

clinica universidad de navarra

Claudio Ronco

Ospedale San Bortolo di Vicenza

\section{Research}

Keywords: TIMP-2*IGFBP7, discontinuation CRRT, renal recovery, NephroCheck

Posted Date: March 30th, 2020 
DOI: https://doi.org/10.21203/rs.2.20260/v2

License: (c) (1) This work is licensed under a Creative Commons Attribution 4.0 International License. Read Full License 


\section{Abstract}

Background Cell cycle arrest biomarkers as TIMP-2*IGFBP7 are elevated in the Acute Kidney Stress and can predict the probability of developing Acute Kidney Injury (AKI). Approximately $25 \%$ of those patients with AKI deteriorate clinically and are unable to maintain adequate homeostasis, eventually requiring continuous renal replacement therapy. However, when clinical improvement occurs, the ideal mode of weaning patients from CRRT is an unmet medical need. Methods We performed a prospective singlecenter study of AKI patients treated with CRRT between October 2017 to April 2019 in a multidisciplinary ICU of an Italian hospital. All patients admitted to ICU requiring CRRT were enrolled. Urine samples for measuring urinary TIMP-2*IGFBP7 levels were collected immediately upon enrollment and at the moment when CRRT was discontinued. The primary endpoint was independence from RRT for at least 7 days after CRRT discontinuation. Renal recovery, which was defined as serum creatinine (SCr) level $<1.5$ times the baseline value at ICU discharge or day 28 , was the secondary endpoint. Results 73 patients were enrolled of whom 45 patients effectively discontinued CRRT (61.6\%). The patients with a TIMP-2*IGFBP7 concentration $>2(\mathrm{ng} / \mathrm{ml}) 2 / 1000$ at enrollment were longer CRRT-dependent. The ROC-AUC values for the prediction of successful discontinuation with TIMP-2*IGFBP7 concentrations at enrollment, at discontinuation of CRRT and with the final model were $0.828,0.814$ and 0.882 , respectively. The risk for CRRT discontinuation failure was nearly 5 times higher patients with a positive biomarker at CRRT discontinuation (OR 4.879, $\mathrm{P}=0.043$ ), and 3.5 times higher in patients with a TIMP-2*IGFBP7 concentration $>2(\mathrm{ng} / \mathrm{ml}) 2 / 1000$ at patient enrollment (OR 3.515, $\mathrm{P}=0.016)$. Multivariate Cox regression analysis showed a significant association between successful discontinuation of CRRT and TIMP2*IGFBP7-negative patients at CRRT discontinuation (RR 0.436, 95\% $\mathrm{Cl} 0.202-0.939, \mathrm{P}=0.034$ ). KaplanMeier curves revealed that TIMP-2*IGFBP7 concentration $<2(\mathrm{ng} / \mathrm{ml}) 2 / 1000$ at enrollment and TIMP$2 *$ IGFBP7 turning negative were positively related to high renal recovery rate. Conclusions Urinary TIMP2*IGFBP7 can serve as a biomarker for identifying successful discontinuation CRRT and predicting renal recovery in critically ill patients.

\section{Introduction}

Acute kidney injury (AKI) is a common complication in critically ill patients and patients who require renal replacement therapy (RRT) have an increased mortality even when adjusted for severity of disease [1, 2]. Several studies have investigated the optimal timing to start continuous renal replacement therapy (CRRT) $[3,4,5]$ without definitively answering the question. Some studies proposed that biomarkers can be used to predict the development of a severe AKI and need for RRT [6, 7]. However, only a few studies investigated the use of biomarkers to predict successful discontinuation of CRRT [8-11]. Newer biomarkers, TIMP-2*IGFBP7, have been shown to be able to predict long term outcome and renal recovery $[12,13]$.

In daily practice, physicians terminate CRRT because the urine output increases, kidney function recovers, or the demand on the kidney is reduced due to improvement of other organ functions. Previous studies showed that different factors are associated with renal recovery, including less severe organ failure, 
shorter duration of RRT, lower age, higher urine output during CRRT, and decreasing plasma NGAL on the first day of RIFLE-F [8-10,14-20]. However, none of these factors is able to predict successful discontinuation of CRRT. Although it has been shown that biomarkers can predict AKI progression [21, 22], it is unknown whether biomarkers can predict successful discontinuation of CRRT

The prediction of the successful discontinuation of CRRT in critically ill patient might prevent harmful under- and overtreatment and subsequently improve patients' outcome and rational use of health care resources. As none of the clinical variables can reliably forecast successful weaning from CRRT, we investigated in this study whether TIMP-2*IGFBP7 in addition to renal and non-renal parameters can predict successful CRRT weaning.

\section{Methods}

\section{Study design and participants}

This is a single-center prospective observational study. All patients (age $\geq 18$ years old) admitted to ICU requiring CRRT from October 2017 to April 2019 at San Bortolo Hospital (Vicenza, Italy) were enrolled in the study. Medical and surgical ICU patients were enrolled. The initiation and cessation of CRRT were decided by nephrologist. Ethical Commite of San Bortolo Hospital of Vicenza approved this study (protocol number 03/17). The consent to participate is pursuant to Italian laws and the written informed consent was obtained from all participants or their legal representative.

Patients with dialysis requirement before ICU admission, end stage renal disease (ESRD), and patients with chronic kidney disease (CKD) stage 4 were excluded. The primary endpoint was the independence from RRT, IHD (intermittent hemodialysis) or restart of CRRT, for at least 7 days after CRRT discontinuation. Restart of CRRT were decided by nephrologist depending on patients' internal environment and capacity load. Renal recovery, which was defined as serum creatinine ( $\mathrm{SCr}$ ) level $<1.5$ times the baseline value and independence RRT at ICU discharge or day 28 (the treatment time of CRRT did not exceed or reach 28 days), was the secondary endpoint [23]. TIMP-2*IGFBP7 lasting positive was defined as TIMP-2*IGFBP7 concentration $>2(\mathrm{ng} / \mathrm{ml})^{2} / 1000$ at enrollment and TIMP-2*IGFBP7 concentration $>0.3(\mathrm{ng} / \mathrm{ml})^{2} / 1000$ at discontinuation of CRRT.

Blood and urine samples for measuring SCr and urinary TIMP-2*IGFBP7 levels were collected immediately upon enrollment and at the moment when CRRT was stopped. TIMP-2*IGFBP7 results were blinded to clinicians. Blood samples for measuring $\mathrm{SCr}$ concentration were also monitored 7 days after discontinuation and at ICU discharge or at day 28 , whatever occurred first. All clinical data were collected from hospital records, including demographic data, medical history, preadmission creatinine (baseline $\mathrm{SCr}$, defined as creatinine up to six months prior to admission), urine output, preadmission estimated glomerular filtration rate (eGFR) (calculated with CKD-EPI formula), previous kidney disease, reason for ICU admission (post-operative, sepsis, respiratory failure, post cardiac arrest, cardiac failure), cause of AKI 
(defined as toxic, sepsis, primary renal disease, ischemic/other) and other variables. In addition, data on RRT, CRRT restart, ICU discharge, and death were recorded.

SCr was measured by enzymatic method with an automatic analyzer (Dimension Vista, Siemens healthcare, Tarrytown, NY, USA). TIMP-2*IGFBP7 levels in the urine were measured by using NephroCheck ${ }^{\circledR}$ (ASTUTE140 meter, Astute Medical, San Diego, CA, USA) approved by the Food and Drug Administration (FDA). TIMP-2*IGFBP7 concentration $>0.3(\mathrm{ng} / \mathrm{ml})^{2} / 1000$ is positive [24].

Estimated glomerular filtration rate (eGFR) was determined by using the Chronic Kidney Disease Epidemiology Collaboration (CKD-EPI) equation. Baseline SCr was determined by the concentration obtained up to six months before hospital admission or at ICU admission in critically ill patients when previous values were not available.

\section{Sample size calculation}

Estimation of sample size is based on preliminary data obtained in a pilot study performed in 77 patients with CRRT requirements in ICU. Estimations performed by using sample size calculators for designing clinical research (www.sample-size.net), assuming an error alpha of 0.05 and a power of $90 \%$, rendered a sample size of 67 patients.

\section{Statistical analysis}

SPSS 20.0 statistical software IBM (NY, USA) was used. Variables were tested for normal distribution using the Kolmogorov-Smirnov test. Normally distributed variables are expressed as mean (standard deviation), non-normally distributed variables as interquartile ranges [P25, P75], and categorical data as number and percentage. Unpaired Student's t-test, Mann-Whitney-U test or Chi-square test was used, where appropriate. Statistical significance was defined as $p<0.05$.

Mann-Whitney test was used for two-group comparison. Categorical variables were compared between groups using Fisher exact test or the chi-square test. The sensitivity and specificity of TIMP-2*IGFBP7 concentrations at enrollment and discontinuation of CRRT for successfully discontinue CRRT were evaluated using the receiver operating characteristic (ROC) curve and area under the curve. Logistic regression analysis was used to analyze related risk factors that influenced discontinuation CRRT. Cox multifactor regression analysis was used to analyze the relationship between all risk factors and successful discontinuation CRRT. Multicollinearity was checked with a maximum variance inflation factor (VIF) of 10. The Kaplan-Meier curves were plotted to assess the renal recovery. Finally, all P-values were two-sided and a P-value $<0.05$ was considered statistically significant.

\section{Results}




\section{General data and outcomes}

From October 2017 to April 2019, a total of 136 patients were screened and 73 patients were enrolled in the study (Figure 1). Baseline characteristics of the included patients are listed in Table 1. Admission diagnoses of the patients are summarized in Table 1. There are no differences regarding TIMP-2*IGFBP7 levels at enrollment and at CRRT discontinuation between the patients of different pathogeny. 45 patients were successfully weaned from CRRT (61.6\%), and 34 patients had renal recovery (46.6\%) (Figure 1). $20.5 \%$ of cohort died during their ICU stay.

\section{Identification of successful CRRT discontinuation}

There were no differences regarding different clinical and laboratory variables, including mean arterial blood pressure, PCT, and lactate, between the successful and unsuccessful CRRT discontinuation groups (Table 2). However, the TIMP-2*IGFBP7 levels at enrollment and at CRRT discontinuation were significantly lower in patients in which CRRT discontinuation was successful, and urine output was significantly increased in this patient group compared to patients in which CRRT discontinuation failed (Table 2). The CRRT treatment duration was significantly longer in patients who had a TIMP-2*IGFBP7 concentration $>2(\mathrm{ng} / \mathrm{ml})^{2} / 1000$ at enrollment $(9.93 \pm 1.59$ days versus $5.43 \pm 0.66$ days, $P=0.013)$.

The successful CRRT discontinuation more likely in patients with a negative TIMP-2*IGFBP7 at enrollment (82.4\%) compared to TIMP-2*IGFBP7-positive patients (52.9\%; $\mathrm{P}=0.057)$ (Table 3). The successful discontinuation $(\mathrm{p}=0.001)$ and renal recovery rate $(\mathrm{p}=0.009)$ was significantly higher in TIMP2*IGFBP7-negative patients at CRRT discontinuation compared to TIMP-2*IGFBP7-positive patients at this time point (Table 3). Patients with a TIMP-2*IGFBP7 level $<2(\mathrm{ng} / \mathrm{ml})^{2} / 1000$ at enrollment had a significantly higher successful CRRT discontinuation rate compared to patients with TIMP-2*IGFBP7 levels $>2(\mathrm{ng} / \mathrm{ml})^{2} / 1000$ at enrollment $(72.5 \%$ versus $42.9 \% ; p=0.027)$ (Table 3$)$. The CRRT treatment time was significant longer in patients with a TIMP-2*IGFBP7 concentration $>2(\mathrm{ng} / \mathrm{ml})^{2} / 1000$ at enrollment than patients with a TIMP-2*IGFBP7 level $<2(\mathrm{ng} / \mathrm{ml})^{2} / 1000$ at enrollment $(9.93 \pm 1.59$ days VS. $5.43 \pm 0.66$ days, $\mathrm{P}=0.013)$.

In univariate regression analysis, we found a significant association between successful discontinuation of CRRT and TIMP-2*IGFBP7-negative at CRRT stop, and TIMP-2*IGFBP7 concentration <2 $(\mathrm{ng} / \mathrm{ml})^{2} / 1000$ at enrollment.

When biomarker levels were measured at CRRT discontinuation, the risk of unsuccessful CRRT discontinuation was nearly 5 times higher in TIMP-2*IGFBP7-positive patients compared to TIMP2*IGFBP7-negative patients (OR 4.879, 95\% Cl 1.055-22.565, P=0.043), whereas the unsuccessful CRRT discontinuation was 3.5 times higher in patients with a TIMP-2*IGFBP7 concentration $>2(\mathrm{ng} / \mathrm{ml})^{2} / 1000$ compared to patients with a TIMP-2*IGFBP7 concentration $<2(\mathrm{ng} / \mathrm{ml})^{2} / 1000$ patients $(\mathrm{OR} 3.515,95 \% \mathrm{Cl}$ 1.267-9.755, $\mathrm{P}=0.016$ ) when measured at enrollment. 
When we substituted TIMP-2*IGFBP7-negative at CRRT stop and TIMP-2*IGFBP7 concentration <2 $(\mathrm{ng} / \mathrm{ml})^{2} / 1000$ at enrollment into the multivariate Cox regression formula, we found a significant association between successful discontinuation of CRRT and TIMP-2*IGFBP7-negative at CRRT stop (RR $0.436,95 \% \mathrm{Cl}$ 0.202-0.939, $\mathrm{P}=0.034)$.

There was no multiple collinearity in our model by calculating the variance inflation factor (VIF) cut-off below 10.

Among the 29 patients, who had an urine output less than $400 \mathrm{ml}$ at the moment when CRRT was stopped, successful CRRT discontinuation rate increased form $0 \%$ in biomarker-positive at CRRT discontinuation to $88.9 \%$ in biomarker-negative patients $(P=0.000)$.

The AUC of the ROC curves for the TIMP-2*IGFBP7 concentrations at enrollment and discontinuation of CRRT for successful discontinuation of CRRT were 0.828 (95\% Cl 0.670-0.986; $p=0.003$ ) and 0.814 (95\% $\mathrm{Cl} 0.658-0.969 ; \mathrm{p}=0.005)$ (Figure 2), with an optimal cut-off of $1.73(\mathrm{ng} / \mathrm{ml})^{2} / 1000$ and 0.90 $(\mathrm{ng} / \mathrm{ml})^{2} / 1000$, sensitivity 0.89 and 0.56 , specificity 0.68 and 0.97 , positive predictive value of $80.0 \%$ and $89.2 \%$, and negative predictive value of $60.6 \%$ and $85.7 \%$, respectively. The AUC of the ROC curve of the final model, including TIMP-2*IGFBP7 concentrations at enrollment and discontinuation of CRRT, was $0.882(95 \% \mathrm{Cl} 0.720-0.998, \mathrm{p}=0.001)$.

\section{Identification of renal recovery}

Patients in the renal recovery group had significantly lower TIMP-2*IGFBP7 levels at enrollment and at CRRT discontinuation as well as a significantly increased urine output compared to the patients in the non-recovery groups (Table 4). There were no differences regarding the other parameters between the recovery and non-recovery group (Table 4 ).

A non-statistically significant trend towards greater renal recovery in TIMP-2*IGFBP7 negative patients at enrolment. Renal recovery rate significantly increased from $21.4 \%$ in patients with a TIMP-2*IGFBP7 concentration $>2(\mathrm{ng} / \mathrm{ml})^{2} / 1000$ at enrollment to $62.5 \%$ in patients with a TIMP-2*IGFBP7 concentration $<2(\mathrm{ng} / \mathrm{ml})^{2} / 1000(\mathrm{P}=0.003)$, and from $44.4 \%$ in biomarker-positive patients at CRRT discontinuation to $69.2 \%$ in biomarker-negative patients $(P=0.009)$ (Table 3 ).

TIMP-2*IGFBP7 values were significantly higher in non-renal recovery patients compared to patients with renal recovery at enrollment and at the discontinuation of CRRT (Table 4).

The risk of non-renal recovery was 6 times higher when TIMP-2*IGFBP7 concentrations were $>2$ $(\mathrm{ng} / \mathrm{ml})^{2} / 1000$ at enrollment compared to TIMP-2*IGFBP7 concentrations $<2(\mathrm{ng} / \mathrm{ml})^{2} / 1000$ patients (OR $6.111,95 \% \mathrm{Cl} 2.021-18.481, P=0.001)$. By calculating the VIF cut-off below 10 , there was no multiple collinearity between TIMP-2*IGFBP7 concentration at enrollment and at discontinuation of CRRT. 
Kaplan-Meier curves revealed that TIMP-2*IGFBP7 concentration $<2(\mathrm{ng} / \mathrm{ml})^{2} / 1000$ at enrollment and TIMP-2*IGFBP7 turning negative were positively related to high renal recovery rate (Figure 3 ).

\section{Discussion}

The TIMP-2*IGFBP7 has been validated in previous cohorts as a potential biomarker in the detection of AKI risk [24-27]. To our knowledge, this is the first study that describes the role of TIMP-2*IGFBP7 in identifying effective discontinuation of CRRT and renal recovery in critically ill patients.

Over the last decades, there has been considerable progress in the development of biomarker utilization for AKI, including risk assessment, diagnosis, prognosis and management. Urinary TIMP-2 and IGFBP7 are novel biomarkers of cell cycle arrest, which may detect the stress of renal epithelial cells and shutdown function without permanent injury. Urinary TIMP-2*IGFBP7 values can be used as diagnostic test to predict AKI in various clinical settings [28-30] and to predict long-term adverse outcome in AKI patients [12, 31]. In these study [12, 31], all-cause mortality and the need of RRT were evaluated. High TIMP-2*IGFBP7 levels were significantly associated with death or RRT in AKI patients.

Depending on the Kidney Disease Improving Global Outcomes guidelines (KDIGO) on AKI recommended [32] and the $17^{\text {th }}$ Acute Quality Initiative international consensus conference recommended [33], it is clear that the timing of discontinuation depends on the clinical judgment and should be adapted according to the needs and requirements of each patient. Nonetheless, the concept of discontinuation CRRT is more complex, since there are no unified criteria. Our study shows that urinary TIMP-2*IGFBP7 concentrations might be used to predict successful discontinuation of CRRT in critically ill patients. In contrast, patients with TIMP-2*IGFBP7 concentrations $>2(\mathrm{ng} / \mathrm{ml})^{2} / 1000$ at enrollment had a higher risk of unsuccessful discontinuation of CRRT. In line with these results, the authors of the Opal study [27] demonstrated in a post-hoc analysis that patients with a high TIMP-2*IGFBP7 concentration at discontinuation of CRRT had a significantly increased risk of unsuccessful discontinuation. In addition, Meersch $M$ et al. [34] found that a decline of TIMP-2*IGFBP7 concentration was an accurate marker of renal recovery following cardiac surgery, with a ROC-AUC 0.79 (95\% Cl 0.65 0.92). Dewitte A and colleagues [35] investigated whether TIMP-2*IGFBP7 predicted short-term renal recovery or non-recovery within $48 \mathrm{~h}$ in 57 patients with early AKI. The decrease of concentration of urinary maker after $24 \mathrm{~h}$ predicted recovery.

Most clinical variables cannot predict renal recovery and successful weaning from RRT. In a recently published study, urine output, serum and urine creatinine levels were identified as variables that might determine the optimal timing of therapy discontinuation [36]. Urine output is the clinical variable that has the best performance for predicting the successful discontinuation of CRRT [37, 38]. However, urine output can be affected by different factors, including ultrafiltration and diuretics. Therefore, new variables need to be found which can predict renal recovery and successful discontinuation of RRT.

In this study, we found that TIMP-2*IGFBP7-positive was not the time to discontinue CRRT. Patients with TIMP-2*IGFBP7 concentration >2(ng/ml) $2 / 1000$ at enrollment were longer treated with CRRT $(9.93 \pm 1.59$ 
days VS. $5.43 \pm 0.66$ days), suggesting that TIMP-2*IGFBP7 might be able to predict renal recovery and successful weaning from CRRT.

Here, we have found that renal recovery after AKI prior to ICU discharge or 28 days significantly increased in patients who had a TIMP-2*IGFBP7 concentration $<2(\mathrm{ng} / \mathrm{ml})^{2} / 1000$ at the enrollment and were TIMP2*IGFBP7-negative at the time CRRT was discontinued. Both TIMP-2*IGFBP7 concentration $>2$ $(\mathrm{ng} / \mathrm{ml})^{2} / 1000$ at enrollment and TIMP-2*IGFBP7 lasting positive are risk of non-renal recovery. The risk of non-renal recovery was 6 times higher in patients with TIMP-2*IGFBP7 concentration $>2(\mathrm{ng} / \mathrm{ml})^{2} / 1000$ at enrollment. The decrease of the urinary TIMP-2*IGFBP7 levels during the course of the disease could potentially predict renal recovery after AKI.

This study has also some limitations. Firstly, TIMP-2*IGFBP7 is a biomarker to predict the risk of AKI within 12 to 24 hours. While we measured urinary TIMP-2*IGFBP7 concentrations immediately upon enrollment, some patients started CRRT after 24 hours or more, and we did not monitor the changes of marker. Secondly, during the CRRT treatment we did not observe the changes. A persistent rise or fall to be negative may be more sensitive. Building on these reasons, we have implemented sequential measurement of TIMP-2*IGFBP7 and established a nephrology rapid response team to care and manage the process of AKI [39].

\section{Conclusions}

Despite of the limitations, the study provided important knowledge. TIMP-2*IGFBP7 was a potential auxiliary tool to identify effective CRRT discontinuation and predict renal recovery after AKI. This finding further confirmed a biologic link between the marker and clinical disease process. Measurement of these biomarkers may improve the ability of management and caring for critically ill patients under CRRT treatment. Measurement of the levels of tubular damager biomarkers complements the information of predicting renal recovery and disease prognosis. The use of this test may guide clinical decision-making and counseling on treatment plans.

\section{List Of Abbreviations}

AKI, acute kidney injury; RRT, renal replacement therapy; CRRT, continues renal replacement therapy; CKD, chronic kidney disease; ESRD, end stage renal disease; Scr, serum creatinine; eGFR, estimated glomerular filtration rate; ROC-AUC, receiver operating characteristic area under the curve; VIF, variance inflation factor; BMI: body mass index; MAP: mean arterial pressure; Hb: hemoglobin; PCT: procalcitonin; NC: NephroCheck;

\section{Declarations}

\section{Ethics approval and consent to participate}


The protocol was approved by the medical ethics committee of the San Bortolo Hospital and the local ethical committees. Written informed consent was obtained from all participants or their legal representative.

\section{Consent for publication}

All authors read and approved the final manuscript.

\section{Availability of data and materials}

All relevant data are within the paper and its supporting information files. All data are fully available without restriction.

\section{Competing interests}

None.

\section{Funding}

None.

\section{Authors' contributions}

R.C. and G.G. designed the study; B.A., S.S., N.M., X.Y. and F.W. acquired the data; X.Y. and R.C. interpreted the data; X.Y. and M.F. did statistical analysis; X.Y. and Z.A. drafted the manuscript; R.C., B.A., R.S. and G.D. supervised and mentored the study. All authors read and approved the final manuscript.

\section{Acknowledgements}

None.

\section{References}

1. Bucaloiu ID, Kirchner HL, Norfolk ER, et al. Increased risk of death and de novo chronic kidney disease following reversible acute kidney injury. Kidney Int. 2012; 5:477-485.

2. Hoste EA, Bagshaw SM, Bellomo R, et al. Epidemiology of acute kidney injury in critically ill patients: the multinational AKI-EPI study. Intensive Care Med. 2015; 41:1411-1423.

3. Zarbock A, Gerß J, Van Aken H, et al. Early versus late initiation of renal replacement therapy in critically ill patients with acute kidney injury (The ELAIN-Trial): study protocol for a randomized controlled trial. Trials. 2016; 17:148.

4. Gaudry S, Hajage D, Schortgen F, et al. Initiation Strategies for Renal-Replacement Therapy in the Intensive Care Unit. N Engl J Med. 2016; 375:122-33. 
5. Barbar SD, Clere-Jehl R, Bourredjem A, et al. Timing of Renal-Replacement Therapy in Patients with Acute Kidney Injury and Sepsis. N Engl J Med. 2018; 379:1431-1442.

6. de Geus HR, Bakker J, Lesaffre EM, et al. Neutrophil gelatinase-associated lipocalin at ICU admission predicts for acute kidney injury in adult patients. Am J Respir Crit Care Med. 2011; 183:907-914.

7. Bennett $M$, Dent $C L, M a ~ Q$, et al. Urine NGAL predicts severity of acute kidney injury after cardiac surgery: a prospective study. Clin J Am Soc Nephrol. 2008; 3:665-673.

8. Dewitte A, Joannès-Boyau O, Sidobre $\mathrm{C}$, et al. Kinetic eGFR and Novel AKI Biomarkers to Predict Renal Recovery. Clin J Am Soc Nephrol. 2015; 10:1900-1910.

9. Srisawat N, Murugan R, Kellum JA. Repair or progression after AKI: a role for biomarkers? Nephron Clin Pract. 2014; 127:185-189.

10. Srisawat N, Murugan R, Lee M, et al. Plasma neutrophil gelatinase-associated lipocalin predicts recovery from acute kidney injury following community-acquired pneumonia. Kidney Int. 2011; 80:545-552.

11. Stads S, Kant KM, de Jong MFC, et al. Predictors of short-term successful discontinuation of continuous renal replacement therapy: results from a prospective multicentre study. BMC Nephrol. 2019; 20:129.

12. Koyner JL, Shaw AD, Chawla LS, et al. Tissue Inhibitor Metalloproteinase-2 (TIMP-2). IGF-Binding Protein-7 (IGFBP7) Levels Are Associated with Adverse Long-Term Outcomes in Patients with AKI. J Am Soc Nephrol. 2015; 26:1747-1754.

13. Meersch M, Schmidt C, Van Aken H, et al. Urinary TIMP-2 and IGFBP7 as early biomarkers of acute kidney injury and renal recovery following cardiac surgery. PLoS One. 2014; 9:e93460.

14. Lin YF, Ko WJ, Chu TS, et al. The 90-day mortality and the subsequent renal recovery in critically ill surgical patients requiring acute renal replacement therapy. Am J Surg. 2009; 198:325-332.

15. Uchino S, Bellomo R, Morimatsu H, et al. Discontinuation of continuous renal replacement therapy: a post hoc analysis of a prospective multicenter observational study. Crit Care Med. 2009; 37:25762582.

16. Wu VC, Ko WJ, Chang HW, et al. Risk factors of early redialysis after weaning from postoperative acute renal replacement therapy. Intensive Care Med. 2008; 34:101-108.

17. Forni LG, Darmon M, Ostermann M, et al. Renal recovery after acute kidney injury. Intensive Care Med. 2017; 43:855-866.

18. Fröhlich S, Donnelly A, Solymos O, et al. Use of 2-hour creatinine clearance to guide cessation of continuous renal replacement therapy. J Crit Care. 2012; 27:744.e1-5.

19. Gibney RT, Bagshaw SM, Kutsogiannis DJ, et al. When should renal replacement therapy for acute kidney injury be initiated and discontinued? Blood Purif. 2008; 26:473-484.

20. Heise $D$, Gries D, Moerer 0 , et al. Predicting restoration of kidney function during CRRT-free intervals. J Cardiothorac Surg. 2012; 7:6. 
21. Alge JL, Arthur JM. Biomarkers of AKI: a review of mechanistic relevance and potential therapeutic implications. Clin J Am Soc Nephrol.2015; 10:147-155.

22. Xie $Y$, Wang $Q$, Wang $C$, et al. High urinary excretion of kidney injury molecule-1 predicts adverse outcomes in acute kidney injury: a case control study. Crit Care. 2016; 20:286.

23. Kellum JA. How can we define recovery after acute kidney injury? Considerations from epidemiology and clinical trial design. Nephron Clin Pract. 2014; 127:81-88.

24. Vijayan A, Faubel S, Askenazi DJ, et al. Clinical use of the urine biomarker [TIMP-2] [IGFBP7] for acute kidney injury risk assessment. Am J Kidney Dis. 2016; 68:19-28.

25. Kashani K, Al-Khafaji A, Ardiles T, et al. Discovery and validation of cell cycle arrest biomarkers in human acute kidney injury. Crit Care. 2013; 17:R25.

26. Bihorac A, Chawla LS, Shaw AD, et al. Validation of cell-cycle arrest biomarkers for acute kidney injury using clinical adjudication. Am J Respir Crit Care Med. 2014; 189: 932-939.

27. Hoste EA, McCullough PA, Kashani K, et al. Derivation and validation of cutoffs for clinical use of cell cycle arrest biomarkers. Nephrol Dial Transplant. 2014; 29: 2054-2061.

28. Wetz AJ, Richardt EM, Wand S, et al. Quantification of urinary TIMP-2 and IGFBP-7: an adequate diagnostic test to predict acute kidney injury after cardiac surgery? Crit Care. 2015; 19:3.

29. Levante C, Ferrari F, Manenti C, et al. Routine adoption of TIMP2 and IGFBP7 biomarkers in cardiac surgery for early identification of acute kidney injury. Int J Artif Organs. 2017; 40:714-718.

30. Pajenda S, Ilhan-Mutlu A, Preusser M, et al. NephroCheck data compared to serum creatinine in various clinical settings. BMC Nephrol. 2015; 16:206.

31. Xie Y, Ankawi G, Yang B, et al. Tissue inhibitor metalloproteinase-2 (TIMP-2) • IGF-binding protein-7 (IGFBP7) levels are associated with adverse outcomes in patients in the intensive care unit with acute kidney injury. Kidney Int. 2019; 95:1486-1493.

32. KDIGO Clinical Practice Guideline for Acute Kidney Injury. 2012. http://kdigo.org/guidelines/acutekidney-injury/

33. Ostermann M, Joannidis $M$, Pani A, et al. Patient selection and timing of continuous renal replacement therapy. Blood Purif. 2016; 42:224-237.

34. Meersch M, Schmidt C, Van Aken H, et al. Urinary TIMP-2 and IGFBP7 as early biomarkers of acute kidney injury and renal recovery following cardiac surgery. PLoS One. 2014; 9:e93460.

35. Dewitte A, Joannès-Boyau O, Sidobre $C$, et al. Kinetic eGFR and Novel AKI Biomarkers to Predict Renal Recovery. Clin J Am Soc Nephrol. 2015; 10:1900-1910.

36. Romero-González G, Lorenzin A, Neri M, et al. Discontinuation of Continuous Renal Replacement Therapy and Dialysis Dependence. Contrib Nephrol. 2018; 194:118-125.

37. Uchino S, Bellomo R, Morimatsu H, et al. Discontinuation of continuous renal replacement therapy: a post hoc analysis of a prospective multicenter observational study. Crit Care. 2009; 37:2576-2582.

38. Katayama S, Uchino S, Uji M, et al. Factors predicting successful discontinuation of continuous renal replacement therapy. Anaesth Intensive Care. 2016; 44:453-457. 


\section{Tables}

Table 1 Baseline characteristics and Diagnoses for ICU admission

\begin{tabular}{|c|c|c|c|c|}
\hline Parameter & Patients & Successful discontinuation & Unsuccessful discontinuation & $\bar{P}$ \\
\hline Number, \% & 73 & $45,61.6 \%$ & $28,38.4 \%$ & \\
\hline Age (years) & $62.07 \pm 17.66$ & $63.33 \pm 17.43$ & $60.04 \pm 18.16$ & 0.442 \\
\hline Sex (male, \%) & $42,57.5 \%$ & $25,55.6 \%$ & $17,60.7 \%$ & 0.808 \\
\hline BMI $\left(\mathrm{kg} / \mathrm{m}^{2}\right)$ & $27.34 \pm 8.29$ & $28.90 \pm 8.76$ & $25.15 \pm 7.23$ & 0.124 \\
\hline Hypertension & $31,42.5 \%$ & $17,17.8 \%$ & $14,50.0 \%$ & 0.338 \\
\hline Diabetes & $31,42.5 \%$ & $19,42.2 \%$ & $12,42.9 \%$ & 1.000 \\
\hline CKD & $29,39.7 \%$ & $17,37.8 \%$ & $12,42.8 \%$ & 0.612 \\
\hline MAP (mmHg) & $80.07 \pm 23.45$ & $80.93 \pm 25.20$ & $78.84 \pm 21.33$ & 0.770 \\
\hline ER $\left(\mathrm{ml} / \mathrm{min} / 1.73 \mathrm{~m}^{2}\right)$ & $73.44 \pm 33.59$ & $74.36 \pm 30.73$ & $72.00 \pm 38.25$ & 0.786 \\
\hline $\mathrm{SCr}(\mathrm{mg} / \mathrm{dl})$ & $0.95(0.731 .43)$ & $0.9(0.731 .31)$ & $0.99(0.771 .87)$ & 0.555 \\
\hline Sepsis & 29 & $18,40 \%$ & $11,39.3 \%$ & \multirow[t]{7}{*}{0.110} \\
\hline Trauma & 6 & $6,13.3 \%$ & 0 & \\
\hline Surgery & 4 & $2,4.4 \%$ & $2,7.1 \%$ & \\
\hline Neurological & 4 & $1,2.2 \%$ & $3,10.7 \%$ & \\
\hline Cardiovascular & 11 & $7,15.6 \%$ & $4,14.3 \%$ & \\
\hline Respiratory & 4 & $4,8.9 \%$ & 0 & \\
\hline Others & 15 & $7,15.6 \%$ & $8,28.6 \%$ & \\
\hline
\end{tabular}

BMI: body mass index; CKD: chronic kidney disease; MAP: mean arterial pressure; eGFR: estimated glomerular filtration rate; $\mathrm{SCr}$ : serum creatinine, $\mathrm{mg} / \mathrm{dL}$ to $\mu \mathrm{mol} / \mathrm{L}, \times 88.4$.

Table 2 Comparison between successful CRRT discontinuation patients and unsuccessful CRRT discontinuation patients

\begin{tabular}{|c|c|c|c|}
\hline Parameter & Successful discontinuation & Unsuccessful discontinuation & P \\
\hline MAP start CRRT (mmHg) & $78.19 \pm 15.14$ & $71.32 \pm 14.42$ & 0.130 \\
\hline Scr start CRRT (mg/dl) & $2.38(1.46,4.09)$ & $2.20(1.33,3.80)$ & 0.821 \\
\hline Urine output start CRRT (ml) & $525.00(41.25,1277.50)$ & $140.00(27.50,1147.50)$ & 0.590 \\
\hline Hb start CRRT (g/dl) & $109.00(91.50,118.00)$ & $101.00(87.00,117.00)$ & 0.300 \\
\hline Bilirubin start CRRT (mg/dl) & $0.80(0.60,1.90)$ & $0.90(0.60,3.10)$ & 0.428 \\
\hline PCT start CRRT (g/l) & $2.86(0.62,54.45)$ & $4.76(0.75,42.63)$ & 0.898 \\
\hline Lactate start CRRT (mmol/l) & $2.70(1.70,5.50)$ & $3.65(1.40,12.05)$ & 0.713 \\
\hline NC enrollment & $1.68 \pm 2.15$ & $4.02 \pm 3.24$ & 0.002 \\
\hline NC discontinue CRRT & $0.16(0.06,0.47)$ & $1.41(0.27,4.74)$ & 0.001 \\
\hline Urine output discontinue CRRT (mL) & $1500.00(450.00,3210.00)$ & $300.00(92.50,600.00)$ & $<0.0001$ \\
\hline Hb discontinue CRRT (g/dl) & $96.00(90.50,104.50)$ & $92.50(81.00,108.75)$ & 0.321 \\
\hline Bilirubin discontinue CRRT (mg/dl) & $1.70(0.55,2.88)$ & $2.00(0.70,3.25)$ & 0.396 \\
\hline PCT discontinue CRRT (g/l) & $2.69(0.88,18.68)$ & $4.28(1.32,8.08)$ & 0.678 \\
\hline Lactate discontinue CRRT (mmol/l) & $1.30(1.10,1.70)$ & $1.90(0.83,6.83)$ & 0.187 \\
\hline CRRT treatment time (days) & $5.00(3.00,11.00)$ & $4.00(2.25,13.00)$ & 0.973 \\
\hline
\end{tabular}

MAP: mean arterial pressure; SCr: serum creatinine, $\mathrm{mg} / \mathrm{dL}$ to $\mu \mathrm{mol} / \mathrm{L}, \times 88.4$; $\mathrm{Hb}$ : hemoglobin; PCT: procalcitonin; NC: NephroCheck; CRRT: continuous renal replacement.

Table 3 TIMP-2*IGFBP7 predicted successful CRRT discontinuation and renal recovery 


\begin{tabular}{|c|c|c|c|c|c|c|c|c|c|}
\hline Variable & $\begin{array}{c}\text { NC (+) } \\
\text { enrollment }\end{array}$ & $\begin{array}{c}\text { NC (-) } \\
\text { enrollment }\end{array}$ & $\mathrm{P}$ & $\begin{array}{c}\text { NC >2 } \\
\text { enrollment }\end{array}$ & $\begin{array}{c}\text { NC <2 } \\
\text { enrollment }\end{array}$ & $\begin{array}{c}\text { P } \\
\text { NC }\end{array}$ & $\begin{array}{c}\text { NC } \\
\text { stop } \\
\text { CRRT }\end{array}$ & $\begin{array}{c}\text { P } \\
\text { stop } \\
\text { CRRT }\end{array}$ & \\
\hline $\begin{array}{c}\text { Successful } \\
\text { discontinuation }\end{array}$ & $52.9 \%$ & $82.4 \%$ & 0.057 & $42.9 \%$ & $72.5 \%$ & 0.027 & $61.1 \%$ & $88.5 \%$ & 0.001 \\
\hline Renal recovery & $41.2 \%$ & $58.8 \%$ & 0.418 & $21.4 \%$ & $62.5 \%$ & 0.003 & $44.4 \%$ & $69.2 \%$ & 0.009 \\
\hline
\end{tabular}

NC: NephroCheck; NC (+): NephroCheck concentration $>0.3(\mathrm{ng} / \mathrm{ml})^{2} / 1000$; CRRT: continuous renal replacement.

Table 4 Comparison between renal recovery patients and non-renal recovery patients

\begin{tabular}{|c|c|c|c|}
\hline Parameter & Renal recovery & Non-renal recovery & P \\
\hline Number, \% & $34,46.6 \%$ & $39,53.4 \%$ & \\
\hline Age (years) & $60.88 \pm 19.82$ & $63.10 \pm 15.74$ & 0.596 \\
\hline Sex (male, \%) & $15,44.1 \%$ & $16,41 \%$ & 0.816 \\
\hline BMI (kg/m ${ }^{2}$ ) & $28.50 \pm 9.63$ & $26.18 \pm 6.70$ & 0.338 \\
\hline Hypertension & $22,64.7 \%$ & $20,51.3 \%$ & 0.343 \\
\hline Diabetes & $21,61.8 \%$ & $21,53.8 \%$ & 0.636 \\
\hline CKD & $19,55.9 \%$ & $20,51.3 \%$ & 1.000 \\
\hline MAP (mmHg) & $81.09 \pm 25.04$ & $79.13 \pm 22.41$ & 0.780 \\
\hline eGFR (ml/min/1.73m $\left.{ }^{2}\right)$ & $75.71 \pm 31.13$ & $71.30 \pm 36.10$ & 0.604 \\
\hline SCr (mg/dl) & $0.92(0.73,1.31)$ & $0.98(0.67,1.64)$ & 0.802 \\
\hline MAP start CRRT (mmHg) & $75.00 \pm 14.56$ & $75.67 \pm 15.84$ & 0.883 \\
\hline Scr start CRRT (mg/dl) & $1.90(1.23,3.92)$ & $2.51(1.57,3.96)$ & 0.238 \\
\hline Urine output start CRRT (ml) & $625.00(95.00,1700.00)$ & $270.00(20.00,845.00)$ & 0.228 \\
\hline Hb start CRRT (g/dl) & $107.00(91.00,120.25)$ & $101.50(91.50,117.25)$ & 0.426 \\
\hline Bilirubin start CRRT (mg/dl) & $1.00(0.60,2.05)$ & $0.80(0.60,2.35)$ & 0.724 \\
\hline PCT start CRRT (g/l) & $4.50(0.89,103.80)$ & $3.29(0.57,46.34)$ & 0.514 \\
\hline Lactate start CRRT (mmol/l) & $2.35(1.45,4.18)$ & $4.40(1.50,13.20)$ & 0.082 \\
\hline NC enrollment & $1.51 \pm 0.37$ & $3.54 \pm 0.51$ & 0.002 \\
\hline NC discontinue CRRT & $0.15(0.06,0.47)$ & $0.36(0.18,1.73)$ & 0.019 \\
\hline Urine output discontinue CRRT (mL) & $1816.76 \pm 264.33$ & $940.00 \pm 180.80$ & 0.008 \\
\hline Hb discontinue CRRT (g/dl) & $98.50(90.50,111.25)$ & $95.00(88.00,103.00)$ & 0.135 \\
\hline Bilirubin discontinue CRRT (mg/dl) & $0.90(0.50,2.40)$ & $2.20(0.75,3.23)$ & 0.141 \\
\hline PCT discontinue CRRT (g/l) & $6.18(1.11,40.53)$ & $1.58(0.65,7.26)$ & 0.064 \\
\hline Lactate discontinue CRRT (mmol/l) & $1.35(1.03,1.60)$ & $1.40(1.00,4.00)$ & 0.265 \\
\hline CRRT treatment time (days) & $5.00(2.75,8.25)$ & $4.00(3.00,13.00)$ & 0.367 \\
\hline
\end{tabular}

BMI: body mass index; CKD: chronic kidney disease; MAP: mean arterial pressure; eGFR: estimated glomerular filtration rate; $\mathrm{SCr}$ : serum creatinine, $\mathrm{mg} / \mathrm{dL}$ to $\mu \mathrm{mol} / \mathrm{L}, \times 88.4$; Hb: hemoglobin; PCT: procalcitonin; NC: NephroCheck; CRRT: continuous renal replacement.

\section{Figures}




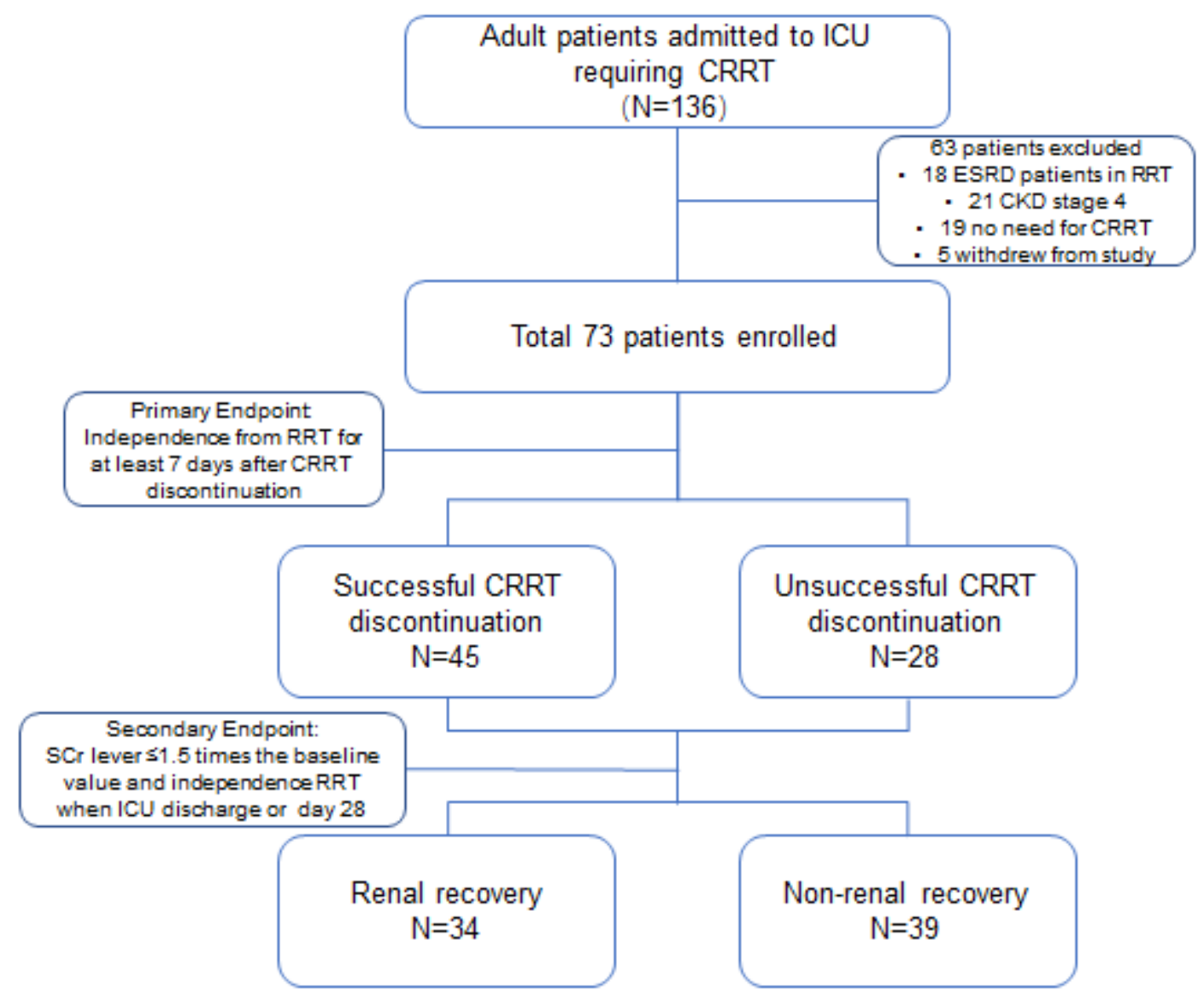

\section{Figure 1}

Flow chart 


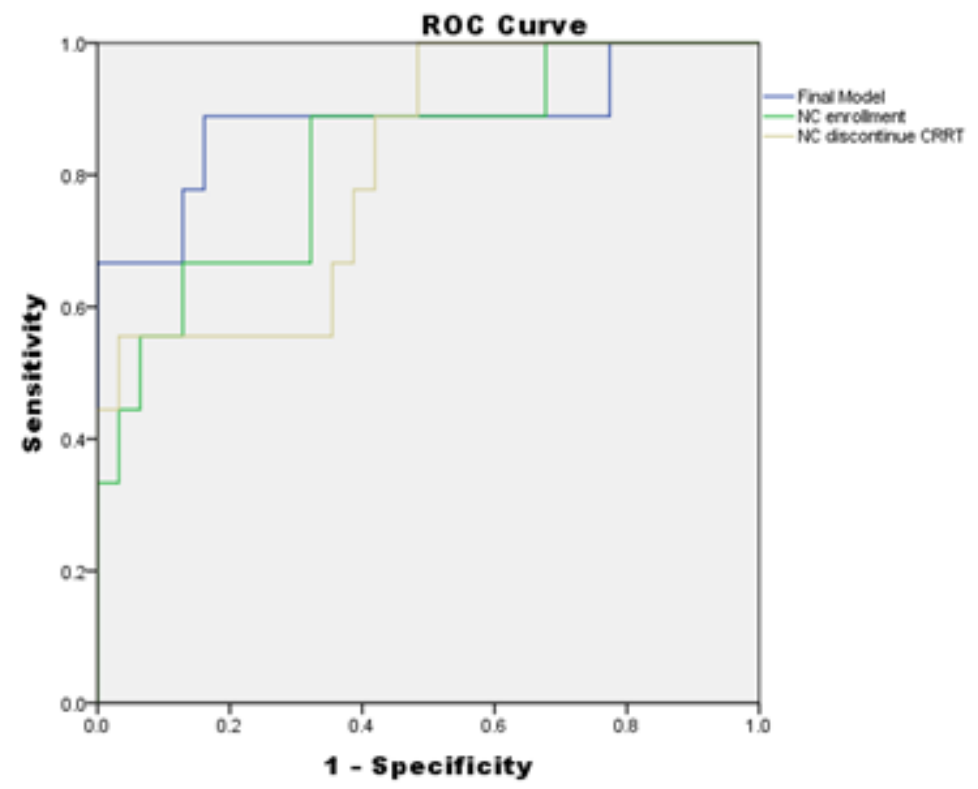

\begin{tabular}{|c|c|c|c|}
\hline & ROC-AUC (95\% Cl) & Sensitivity, Specificity & P \\
\hline NC enrollment & $0.828(0.6700 .986)$ & $88.9 \%, 67.7 \%$ & 0.003 \\
\hline NC discontinue CRRT & $0.814(0.6580 .969)$ & $55.6 \%, 96.8 \%$ & 0.005 \\
\hline Final model & $0.882(0.720-0.998)$ & $88.9 \%, 83.9 \%$ & 0.001 \\
\hline
\end{tabular}

\section{Figure 2}

ROC-AUC values for the prediction of successful CRRT discontinuation
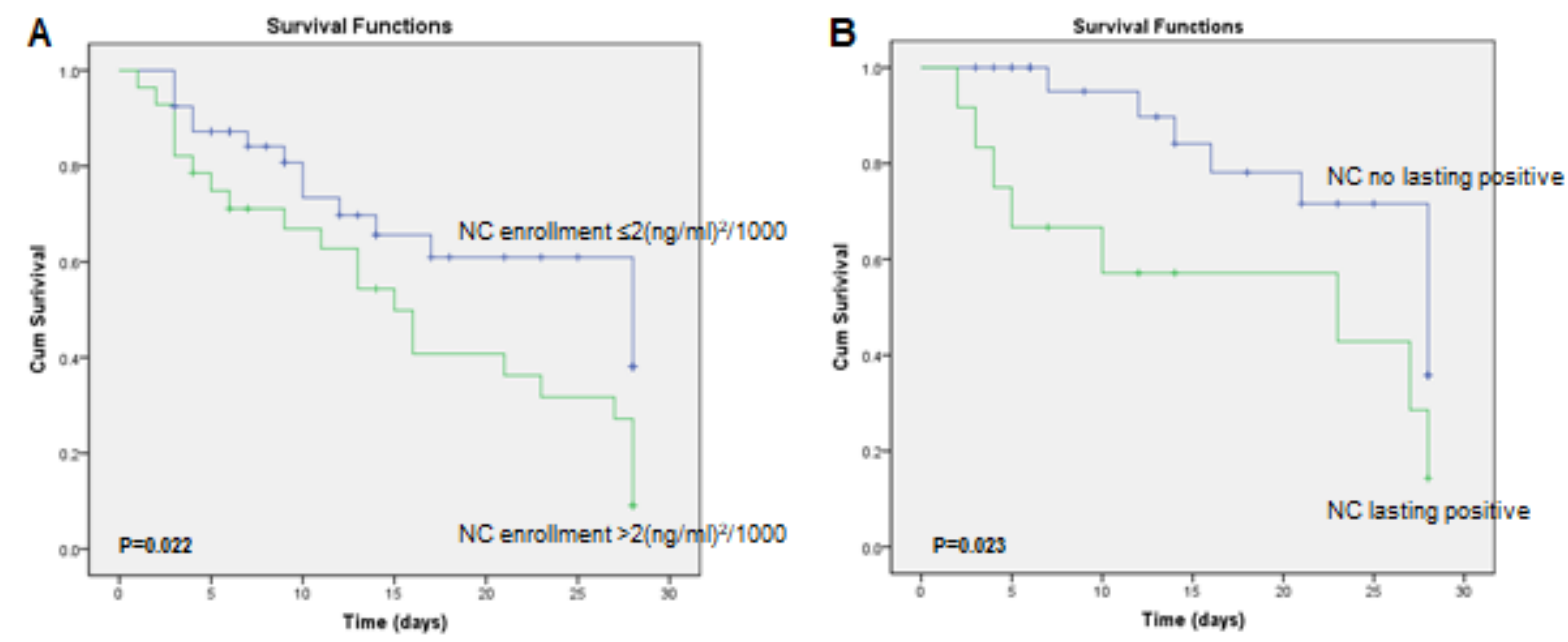

\section{Figure 3}

K-M curves for the survival estimate of renal recovery NC lasting positive: TIMP-2*IGFBP7 concentration $>2(\mathrm{ng} / \mathrm{ml}) 2 / 1000$ at enrollment and TIMP-2*IGFBP7 concentration $>0.3(\mathrm{ng} / \mathrm{ml}) 2 / 1000$ at discontinuation of CRRT 